\title{
Editorial
}

\section{Smart Polymeric Nanocarriers}

\author{
Jianxun Ding, ${ }^{1}$ Yilong Cheng, ${ }^{2}$ Mingqiang Li, ${ }^{3}$ Ruxandra Gref, ${ }^{4}$ and Xuesi Chen ${ }^{1}$ \\ ${ }^{1}$ Key Laboratory of Polymer Ecomaterials, Changchun Institute of Applied Chemistry, Chinese Academy of Sciences, \\ Changchun 130022, China \\ ${ }^{2}$ Department of Bioengineering and Molecular Engineering and Sciences Institute, University of Washington, Seattle, WA 98195, USA \\ ${ }^{3}$ Department of Biomedical Engineering, Columbia University, New York, NY 10027, USA \\ ${ }^{4}$ Institute of Molecular Sciences, CNRS, Université Paris-Sud, Université Paris Saclay, 91400 Orsay, France \\ Correspondence should be addressed to Jianxun Ding; jxding@ciac.ac.cn
}

Received 12 October 2016; Accepted 12 October 2016

Copyright (C) 2016 Jianxun Ding et al. This is an open access article distributed under the Creative Commons Attribution License, which permits unrestricted use, distribution, and reproduction in any medium, provided the original work is properly cited.

Recently, polymeric nanocarriers, including micelles, vesicles, capsules, nanogels, nanospheres, and nanofibers, have attracted more and more attention in the field of drug delivery. Specifically, the development of smart polymeric nanocarriers enables the personalized and on-demand treatments to be highly possible. Their intelligence not only endows the polymeric nanocarriers with the capabilities of identifying diseased tissue or cells and triggering the positive response of cells, but also serves as a switch to achieve directional drug delivery in the lesion sites. The potential advances of smart polymeric nanocarriers bring opportunities as well as challenges for researchers to exploit more desirable nanosystems for controlled drug release.

The purpose of this special issue is to publish high-quality research articles as well as reviews that seek to address recent developments in the preparation, characterization, and application of smart polymeric nanocarriers as well as the relevant prospect on opportunities and challenges.

Polymeric micelles with a core-shell architecture are nanoscopic colloidal aggregates of amphiphilic polymers dispersed in aqueous medium. The inner cores are composed of hydrophobic polymer blocks and serve as drug reservoirs, and the hydrophilic polymer shells endow micelles with water solubility and long blood circulation time in vivo. M. Wang et al. synthesized folate-biotin-pullulan (FBP) through a onepot condensation reaction. FBP self-assembled into micellar nanoparticle with a diameter of $156 \pm 13 \mathrm{~nm}$ and zeta potential of $-5.1 \pm 0.6 \mathrm{mV}$. Doxorubicin (DOX), as a model antitumor drug, was loaded into the FBP micelle, and the drug-loaded micelle was denoted as FBP/DOX. FBP/DOX showed the best tumor-suppressing effect compared with free DOX and its nontargeted counterpart as a result of the folate-receptormediated endocytosis of FBP/DOX. Overall, the targeted polymeric micelles exhibited great potential to selectively deliver antitumor drugs and upregulate the antitumor efficacy.

Polymeric nanogels are three-dimensional (3D) nanosized hydrogels, which are fabricated by the physical or chemical crosslinking of polymers. Nanogels have attracted growing interest in controlled drug delivery because of their tunable chemical and physical structures and considerable stability in vivo. M. Usacheva et al. prepared a calcium-crosslinked dioctyl sodium sulfosuccinate (aerosol OT, AOT)-alginate nanogel loading toluidine blue (TB) for the photodynamic therapy (PDT) of the biofilm-mediated infections of chronic wounds. The TB-loaded nanogel induced effective killing of planktonic $P$. aeruginosa $(3.5 \mathrm{lg}(\mathrm{CFU}))$ and $S$. aureus $(>5 \mathrm{lg}$ $(\mathrm{CFU})$ ) as well as their combined biofilms of $2.8 \mathrm{lg}$ (CFU) for $P$. aeruginosa versus $3.4 \mathrm{lg}(\mathrm{CFU})$ for $S$. aureus. The results demonstrated the effective eradication of complex biofilms of dual bacterial strains by polymeric nanoparticle-mediated PDT.

Nanofibers are generally defined as fibers with diameters less than $1 \mu \mathrm{m}$. Polymeric nanofibers are widely used for in situ drug delivery. H. Zhou et al. constructed a resveratrol(RSV-) loaded electrospun nanofiber of methoxy poly(ethylene glycol)-poly( $\varepsilon$-caprolactone) (mPEG-PCL) block copolymer as a drug carrier. The loading nanofiber (i.e., RSV-NF) exhibited more apoptosis induction and low migration and invasion ability of human U87 glioblastoma cells. Furthermore, the local implantation of RSV-NF greatly enhanced the growth inhibitory effect compared to free RSV. The results 
demonstrated that nanofibers are a promising strategy to improve the effects of antitumor drugs. H. Liu et al. fabricated the silver-plated cotton fabrics (SPCFs) with high electrical conductivity and excellent washing fastness. With the increase of silver-ammonia concentration or dopamine concentration, the surface resistivity of SPCFs decreased and gradually stabilized. The surface resistivity of SPCFs could reach $0.12 \pm 0.02 \Omega$, and the electromagnetic shielding effectiveness (ESE) of SPCFs could reach $58.5 \pm 4.5 \mathrm{~dB}$. The conductive fabrics have a wide range of applications in many fields, such as antibacterial, intelligent textiles, smart garments, electromagnetic shielding, and flexible sensors.

In basic research, many complex multifunctional polymeric nanoplatforms have been designed for smart drug delivery. X. Tang et al. prepared a LY2835219-loaded hyaluronic acid-conjugated chitosan nanoparticle (HACNP/LY) for enhanced antitumor efficacy through the p16-CDK4/6-pRb pathway against human HT29 colon cells. The LY2835219loaded chitosan nanoparticle (CNP/LY) was used as a control. HACNP/LY showed the highest proliferation inhibition efficiency toward HT29 cells. HACNP/LY strengthened the trend further compared to CNP/LY because of the CD44mediated targetability.

J. Ding and coworkers contributed a review article, which summarized the development of polymeric nanocarriers for antineoplastic delivery in the treatment of spinal malignancies and discussed the future prospects of polymeric nanocarrier-based treatment methods. The review article provides a summary of various smart polymeric nanocarriers for controlled drug delivery and enhanced treatment efficacies, with spinal malignancies as a disease model.

In addition to the above polymeric nanocarriers, organicinorganic hybrid nanomaterials, liposome, noisome, emulsion, and so forth, are also exploited for controlled drug delivery and showed good results as well as great potential for clinical applications.

The collection of articles in this special issue highlights the development of nanocarriers, particularly smart polymeric nanocarriers, for selective drug delivery. The guest editors and contributors hope that this thematic issue will inspire the readers and stimulate the advanced researches in the area of controlled drug delivery.

\title{
Acknowledgments
}

The guest editors are grateful to all authors for their highquality contributions to the special issue. They would like to acknowledge all reviewers for their valuable comments on the manuscripts from this special issue.

\author{
Jianxun Ding \\ Yilong Cheng \\ Mingqiang $L i$ \\ Ruxandra Gref \\ Xuesi Chen
}



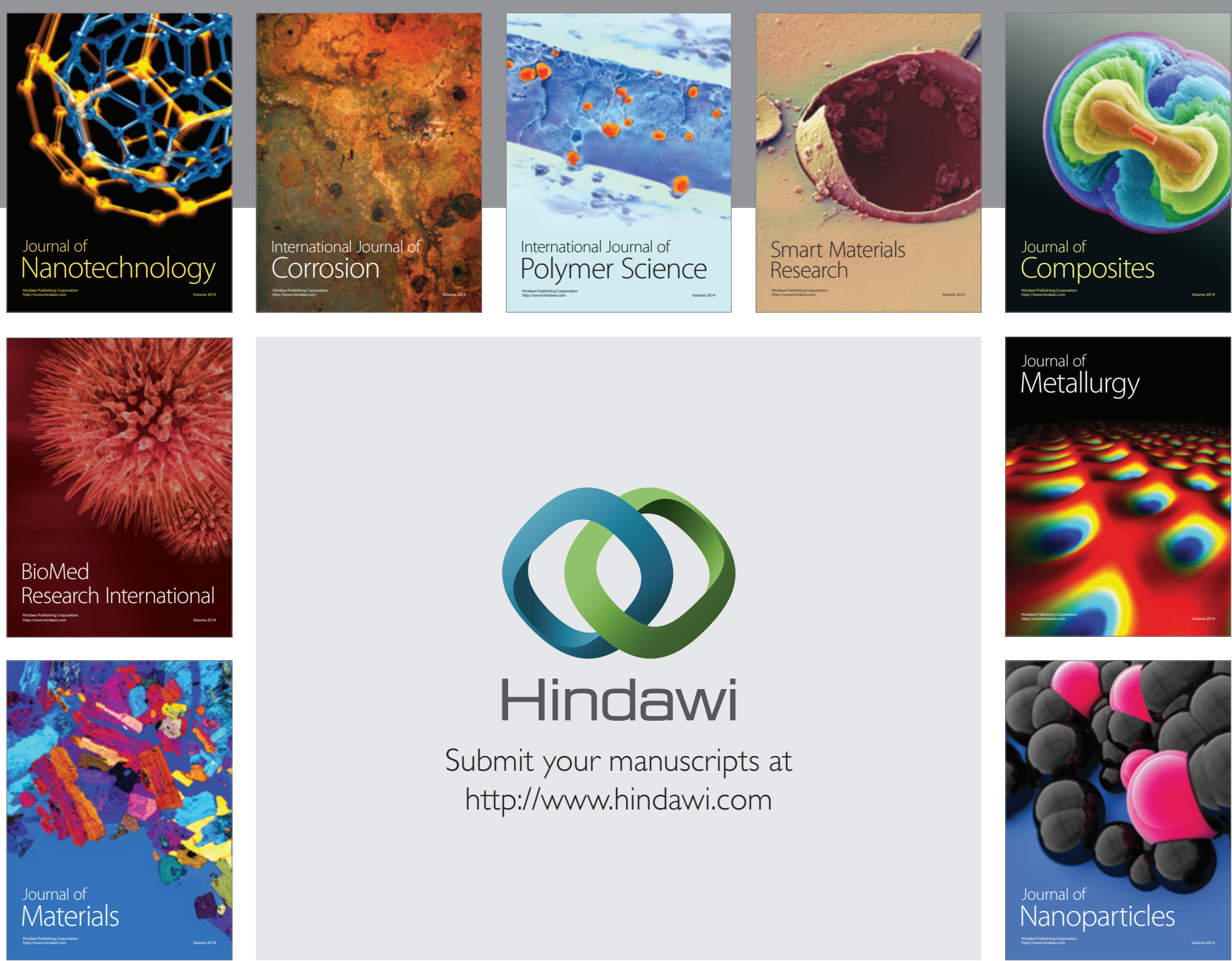

\section{Hindawi}

Submit your manuscripts at

http://www.hindawi.com

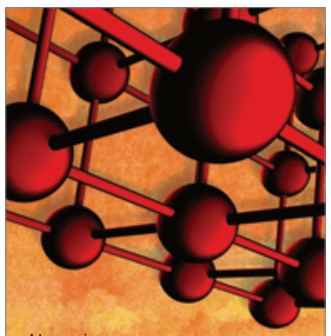

Materials Science and Engineering
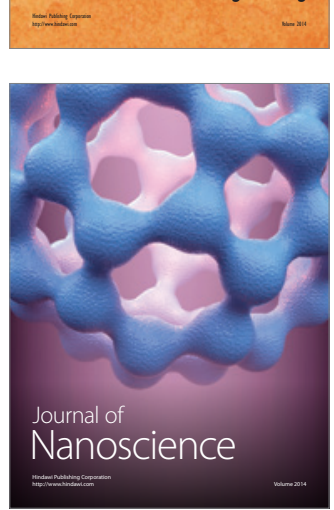
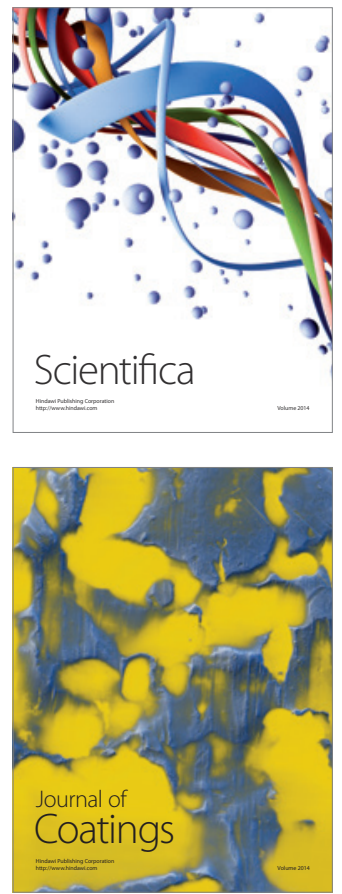
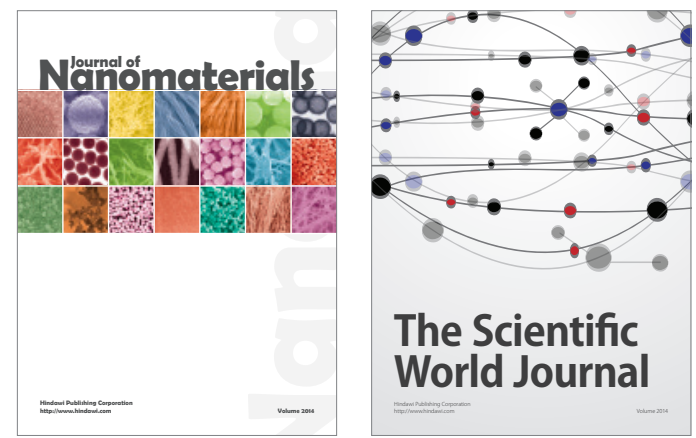

The Scientific World Journal
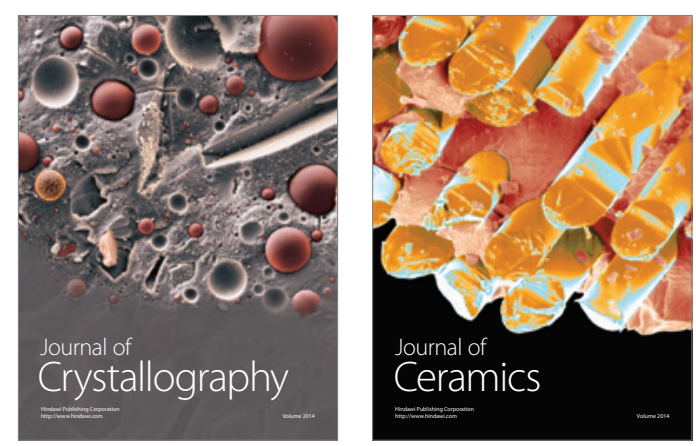
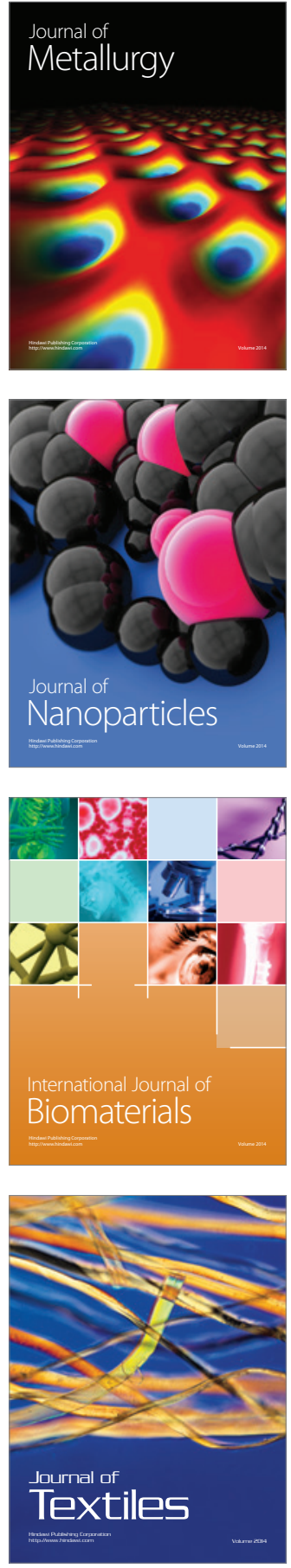